\title{
NOVAS LÓGICAS, RACIONALIDAES E CONTEXTOS NA PREVENÇÃO QUATERNÁRIA
}

\author{
Júlia Maria Guilherme Ribeiro Antunes \\ Médica e Médica Dentista \\ Unidade de Saude Egas Moniz \\ Assistente, Portugal \\ julia@antunes.net
}

Fecha de Recepción: 22 Marzo 2018

Fecha de Admisión: 10 Abril 2018

\section{RESUMO}

A Medicina Preventiva, especialmente nos paises ditos, desenvolvidos, é alvo de interesse crescente não só para as pessoas que procuram a tranquilidade da saúde, obtida atraves de longas cascatas de exames, observações e consultas, mas também e sobretudo para os prestadores de cuidados médicos e a indústria farmacêutica que cada vez facturam mais milhões!

A prevenão quaternária veio nos ultimos anos trazer uma lufada de lúcidez na problemática saúde / doença, e está hoje presente nas varias especialidades médicas, não parando de crescer e encontrando mais e mais adeptos para identificar pessoas em risco de medicalização excessiva, evitando assim danos iatrogénicos.

A prevenção primaria e secundaria apoiadas pela vontade dos individuos e dos profissionais, encontram terreno fértil á conversão de saudáveis em doentes sob o olhar atento das multinacionais farmacêuticas, sendo que, a prevenção destas prevenções cabe sem duvida, á Prevenão Quaternária, interveniente cada vez mais em novas lógicas, racionalidades e contextos da actualidade.

Palavras chaves: práticas de saúde; prevenção quaternária

\section{ABSTRACT}

New logics, rationalities and contexts in quaternary prevention.

The University students are usually well educated, healthy, is a relatively homogeneous socioeconomic and relatively privileged group culture and "status ", being undoubtedly a potential future leadership that can influence with some ease other social groups at different times of the cycle life, taking on the role of change agents. They are also, will start, more motivated to knowledge about behaviors and healthy lifestyles. Show that attitudes towards issues of common health ? Which actors, the itineraries of health and illness that question ? Laymen , doctors, pharmacists, neighbors , friends, family ? Or just wait \& see ? 


\section{NOVAS LÓGICAS, RACIONALIDAES E CONTEXTOS NA PREVENÇÃO QUATERNÁRIA}

Were surveyed by questionnaire 502 college students from Health Sciences. It is an exploratory , descriptive and cross-sectional study which presents some results in this article , that it highlights the correlation of their health practices with the concept quaternary prevention, in which most of the time only involve the technique of wait \& see.

This is opposed to those that shaped the medicalization of our society in a very recent past. We may think of this social group as a motivator for profound changes in the practices of health / disease for next years , applying day by day, the concept and philosophy of quaternary prevention?

Keywords: health practices; quaternary prevention

\section{INTRODUÇÃO}

A Prevenção Quaternaria é uma filosofia nova com potencial para modificar a forma como a nossa sociedade lida com práticas de saude e doença. Assim, "as cascadas diagnosticas» podem ser travadas com o simples «esperar e ver» ou «wait and see» também chamada demora «permitida». As campanhas de prevenção de benefícios duvidosos ou mesmo desnecessárias devem ser alvo criticas como as avaliações de risco genético (Gérvas, 2003).

A prevenção quaternária defende o principio da proporcionalidade (os ganhos devem superar os riscos) e o da precaução (primum non nocere) ou seja, primeiro não lesar (Almeida, 2005).

A prevenção quaternária prima pela ética da negativa isto é simplesmente dizer não à corporação profissional-tecnológico-farmacêutica inclusive à opinião publica (Gérvas 2006 e 2005).

Em 2002, Starfield referiu que, 401\% dos pacientes, que trazem um novo problema de saúde à consulta, melhoram sem que tenha sido formulado um diagnóstico específico. No compasso de espera, o diagnóstico pode ser afinado, reduzindo-se as possibilidades de falsos positivos e de falsos negativos dos testes diagnósticos.

Por estas razões Gérvas $\backslash \&$ Pérez-Fernández (2006) referem "A chave da prevenção quaternária é não iniciar a cascata de exames, não classificar o paciente, não abusar do poder de definir o que é enfermidade, factor de risco e saúde. Há que se resistir tanto à pressão da corporação farmacêutica, tecnológica e profissional como também dos pacientes. Há que se desenvolver e estruturar uma ética negativa, baseada no contrato social implícito que exige do médico o comprimento de sua obrigação mesmo que haja uma demanda insaciável para iniciar a cascata diagnóstica e preventiva desnecessária

Na prática clínica diária a prevenção quaternária cada vez é mandatório estar mais presente, ela limita os abusos de definição de saúde, doença e factores de risco. Exige actualização permanente espírito crítico e sobretudo bom senso.

Em 1966 e 1973, Irving Zola, interessou-se pel analise dos motivos de consulta e verificou que aquilo que numa cultura é um sinal para ir ao médico e que é assim indicador de doença, noutra nem por isso, pode mesmo ser encarado dentro da normalidade.Em 1995, Massé fala do saber leigo sobre a saude e a doença como um sub-sistema cultural com os seus conhecimentos e crenças e também os seus valores, símbolos, concepcões e representações segundo uma lógica própria.As causas de doença, relacionam-se com o quadro de vida do individuo, com a sua rede de suporte social e com o seu universo social mais amplo, a comunidade.A esta epidemiologia sociocultural centrada nos diagnosticos médicos podemos acrescentar uma etno-epidemiologia para estudo e caracterização das categorias diagnosticas leigas e assim as doenças aparecem-nos como construções socioculturais que podem ter tantos significados quanto os contextos em que são definidas.Nesta "nova epidemiologia,"os diagnosticos poderão ser considerados como construtos culturais (Massé,1995). 0 saber leigo sobre saude e doença, contextualiza não só os sintomas, mas também outros elementos como as causas, as circunstâncias, os comportamentos.Para os tecnicos de 
saúde, a probailidade do risco justifica a evicção do comportamento para os leigos nem por sso pois o perigo nem sempre se confirma.

Em 1977, Cassel lembrou que as pessoas têm a sua personalidade, experiência de vida, laços famiiares,cultura, visão do mundo, expectativas de futuro.È com todo este arsenal que enfrentam a doença e desenvolvem as respostas ao sofrimento ao qual são mais sensiveis enqanto os tecnicos percepcinam mais a doença-patologia.

A cultura leiga, está em permanente reconstrução e apresenta um dinamismo na integração dos diversos actores, presentes e concorrentes a uma resposta final.

Assim, os conhecimentos da medicina cotribuem para compreender o significado do adoecer, os tratamentos podem envolver os receitados pelo médico, pelo vizinho, amigo, familiar, as "mesinhas"as medicina alternativas, ou complementares á medicina alopática, numa corrida vertiginosa que as vezes parece paradoxal mas que á luz das representações socias, dos valores, da experiência e tradição acabam por explicar as escolhas que 0 individuo vai fazer.

0 paciente a quem foi prescrito um medicamento na sua privacidade, entra numa linha de "automedicação", isto é, ele vai decidir a quantidade se toma ou não quando toma e outras variaveis que poderão aparecer segundo os casos em questão.Trata-se de utilizar ma poção mágica, receitada por um "feiticeiro do nosso tempo" perfeitamente legitimado : 0 médico.

Em 2001, Fainzang, encontrou explicação para alguns comportamentos segundo a variavel religião. Para catolicos e muculmanos não há direito á interrupção do tratamento, os judeus e protestantes reivindicam -no.A receita arquivada pelos judeus, é deitada fora pelos muculmanos.

A fenomenologia da saude/doença envolve para além do saber leigo, a ciência e arte médica, bem com o sector alternativo. Kleinman (1988) desenvolveu este modelo a que os leigos apelam ao longo das suas vidas, e em que a explicação da doença, os algoritmos diagnosticos e os tratamentos são diferentes.Nalgumas sociedades, o sector alternativos está tão presente quanto o sector médico.A homeopatia, acupunctura, fitoterapia, naturopatia, osteopatia expandiram-se notoriamente desde os anos 50 .

Até ao encontro com um profissional, médico ou alternativo, é no sector leigo que as pessoas vão procurar ajuda, e é a ele que regressam depois de sairem do consultório, aí avaliam resultados e decidir como continuar.Auto-cuidados, cuidados tradicionais, e cuidados alternativos vão-se arranjar e combinar, ao longo do itinerário terapêutico, avaliando-se permanentemente a eficácia que deles se espera (Fassin,1992). È um processo dinâmico, conduzido pelo paciente dependente de lógicas multiplas, é um itinerário terapêutico nos itinerários de saude-doença!

Vamos procurar conhecer, que atitudes desenvolvem os Universitários perante alguns problemas de saúde frequentes no nosso dia a dia.

\section{MATERIAL E MÉTODOS}

Podemos definir este estudo como descritivo, transversal e exploratório acerca do qual se irão apresentar alguns resultados.

A unidade de observação foi "aluno de curso de Ciências da Saúde". Neste caso responderam os alunos dos cursos de Cardiopneumologia, Radiologia, Terapia da Fala e de Fisioterapia, Anatomia Patológica, Citológica e Tanatológica e Prótese Dentária.

Os questionários foram aplicados em sala de aula visando a maior concentração de jovens em ambiente tranquilo no local de reflexão com vista ao seu melhor preenchimento.

Foi realizado um pré-teste a 50 jovens, préviamente, clarificando alguns afinamentos pontuais para 0 formato definitivo. Os questionários foram aplicados na totalidade pela investigadora a 502 alunos. 


\section{NOVAS LÓGICAS, RACIONALIDAES E CONTEXTOS NA PREVENÇÃO QUATERNÁRIA}

0 tratamento dos dados foi efectuado em duas fases correspondendo, ás fases da colheita de dados também duas (2010/2011) este intervalo permitiu redefinir e refletir a problematização em estudo, afinando alguns dos seus objectivos. A base de dados foi encerrada em Fevereiro de 2011.

\section{CARACTERIZAÇÃO DA AMOSTRA}

Esta amostra não é probabilística nem representativa. 0 conjunto de indivíduos do nosso estudo foi escolhida por conveniência.

Se a investigação é do tipo descritivo, exploratório, podem ser utilizadas amostras não aleatórias, uma vez que não se pretende realizar uma análise crítica dos dados apurados, para daí inferir e extrapolar para a população que está a ser objecto de investigação. Os estudos descritivos/exploratórios são desenhados para problemas novos ou pouco estudados nos quais se pretende saber como abordar e estudar os fenómenos em causa,

Como referem as Ciências Sociais "... não podemos esquecer as diferenças existentes entre amostra, estatisticamente e teoricamente representativa. À primeira exige-se que permita definir relações na distribuções dos inquiridos por categorias e a segunda que descubra novas categorias teoricamente pertinentes..." (Silva, 1986)

No quadro que segue descreve-se sucintamente a amostra:

Quadro I

Amostra

\begin{tabular}{|l|c|}
\hline Mulheres & $76,7 \%$ \\
\hline Homens & $21,9 \%$ \\
\hline N/A & $1,4 \%$ \\
\hline
\end{tabular}

Neste estudo as mulheres representam $76,7 \%$ da amostra, contra $21,9 \%$ de homens. Isto é concordante com a frequência feminina cada vez maior do Ensino Superior de Ciências da Saúde. A média de idades femininas e masculinas (18/21) adequa-se com o estatuto de jovem estudante universitário.

\section{TRATAMENTO E ANÁLISE DOS DADOS}

0 tratamento estatístico dos dados foi feito com recurso ao programa de análise SPSS para Windows, versão 15.0 .

Numa primeira fase foram eliminadas todas as respostas incompletas e colectadas as respostas válidas ás quais se aplicaram cálculos de médias, moda, frequências e percentagens.

\section{RESULTADOS}

Dos 502 individuos que responderam ao inquérito, 385 (76,7\%) identificaram-se como mulheres e $110(21,9 \%)$ como homens. Os restantes 7 inquiridos $(1,4 \%)$ não indicaram 0 sexo.

A distribuição etária dos inquiridos localiza-se maioritariamente (69,9\%) entre os 18 e 21 anos, como é natural neste tipo de amostra de jovens universitários.

Quanto ao Estado Civil, a maioria dos inquiridos $(95,4 \%)$ é solteiro, o que se compreende devido às idades dos inquiridos e condição de estudante.

0 local de residência dos inquiridos situa-se maioritariamente $(75,9 \%)$ nos Distritos circundates (Setúbal e Lisboa) ao local de estudo.

Todos os inquiridos são estudantes do Ensino Superior pelo que todos possuem o Ensino 
Secundário completo, mas alguns (28 \%) têm outras licenciaturas (130), mestrados (12) e doutoramento (1), o que começa a ser habitual nos tempos actuais em que os jovens diversificam os cursos, até porque não encontram saída profissional para o curso que completaram.

Quanto a Seguro de Saúde, 261 do total dos inquiridos (53,3 \%) possui Seguro de Saúde

Foi feita uma pergunta ácerca das atitudes perante alguns problemas de saúde (febre, tosse, cansaço, dores no corpo, nervosismo, tristeza, insónias, dores de cabeça, dores de dentes, má digestão, mal da barriga, problemas de pele, gripe, ver mal, dificuldades sexuais ) e sua resolução (esperar que passe, usar tratamentos caseiros, usar medicamentos que conhece, falar com o médico ou farmacêutico ou amigo, vizinho ou familiar) cujos resultados se apresentam no quadro seguinte:

Atitudes dos estudantes universitarios perante problemas de saude frequentes.

\begin{tabular}{|l|c|c|c|c|c|c|}
\cline { 2 - 7 } & $\begin{array}{c}\text { Espera que } \\
\text { passe }\end{array}$ & $\begin{array}{c}\text { Usa } \\
\text { tratamentos } \\
\text { caseiros }\end{array}$ & $\begin{array}{c}\text { Usa medicamentos } \\
\text { que conhece }\end{array}$ & Vai à farmácia & Vai ao médico & $\begin{array}{c}\text { Fala com um } \\
\text { amigo/ vizinho/ } \\
\text { familiar }\end{array}$ \\
\hline 1) Febre & $10 \%$ & $5 \%$ & $61 \%$ & $9 \%$ & $14 \%$ & $1 \%$ \\
\hline 2) Tosse & $23 \%$ & $19 \%$ & $42 \%$ & $14 \%$ & $2 \%$ & $0 \%$ \\
\hline 3) Cansaço & $83 \%$ & $7 \%$ & $3 \%$ & $1 \%$ & $5 \%$ & $1 \%$ \\
\hline 4) Dores no corpo. & $60 \%$ & $7 \%$ & $22 \%$ & $3 \%$ & $7 \%$ & $0 \%$ \\
\hline 5) Nervosismo & $76 \%$ & $6 \%$ & $8 \%$ & $1 \%$ & $5 \%$ & $5 \%$ \\
\hline 6) Tristeza & $78 \%$ & $1 \%$ & $1 \%$ & $0 \%$ & $4 \%$ & $16 \%$ \\
\hline 7) Insónias & $70 \%$ & $7 \%$ & $8 \%$ & $5 \%$ & $7 \%$ & $3 \%$ \\
\hline 8) Dores de cabeça & $21 \%$ & $3 \%$ & $68 \%$ & $5 \%$ & $3 \%$ & $0 \%$ \\
\hline 9) Dores de dentes & $8 \%$ & $2 \%$ & $43 \%$ & $7 \%$ & $39 \%$ & $1 \%$ \\
\hline 10) Má digestão & $37 \%$ & $17 \%$ & $30 \%$ & $7 \%$ & $9 \%$ & $1 \%$ \\
\hline 11) Mal da barriga & & $10 \%$ & $35 \%$ & $6 \%$ & $8 \%$ & $1 \%$ \\
\hline 12) Problemas de pele & $9 \%$ & $2 \%$ & $10 \%$ & $8 \%$ & $70 \%$ & $1 \%$ \\
\hline 13) Gripe / Constipação & $9 \%$ & $4 \%$ & $48 \%$ & $11 \%$ & $27 \%$ & $1 \%$ \\
\hline 14) Ver mal & $4 \%$ & $0 \%$ & $1 \%$ & $1 \%$ & $93 \%$ & $1 \%$ \\
\hline 15) Difículdades sexuais & $17 \%$ & $2 \%$ & $1 \%$ & $2 \%$ & $62 \%$ & $17 \%$ \\
\hline
\end{tabular}

\section{DISCUSSÃO}

Como estudo exploratório não the podemos conferir o rigor necessário para extrapolar conclusões para 0 universo correspondente a este grupo etário. No entanto para esta amostra de alunos universitários do primeiro ano de cursos da área de Ciências da Saúde, podemos observar que curiosamente e exceptuando dores de dentes, problemas de pele, gripe/constipação, ver mal, dificuldades sexuais, em que consultam o médico preferencialmente ao farmacêutico, na maior parte dos problemas apresentados, há a atitude da "espera" ou "wait \& see", nalguns casos como na febre, na tosse, dores no corpo, dores de cabeça/dentes,má digestão, mal da barriga, gripe/constipação, usam medicamentos que já conhecem, provavelmente prescritos para a mesma finalidade anteriormente, tratando-se aqui de automedicação responsável, que se tem tentado desenvolver com politicas para aí direcionadas, nos ultimos anos tendo em vista as actuais dificuldades económicas das nossas sociedades. Tambem curiosamente, os tratamentos caseiros, aparecem na tosse, má digestão e mal de barriga, deixando supor velhas ilustrações do imaginário leigo, que necessitam mais estudo para as decifrar. Os amigos, vizinhos e familiares são consultados na tristeza, tavez como "desabafo" e nos problemas sexuais que são da esfera intima. 


\section{NOVAS LÓGICAS, RACIONALIDAES E CONTEXTOS NA PREVENÇÃO QUATERNÁRIA}

\section{CONCLUSÕES}

No seu conjunto, parecem co-existir harmonia e adequação de atitudes tempradas com bom senso, nestes jovens universitários, que tavez sem saberem praticam principios de prevenção quaternária tão defendidos nalguns circulos do saber, como forma de limitar os problemas de medicalização, iatrogenia, interacçôes medicamentosas presentes na nossa sociedade.Serão futuramente estes os interlucotores que procuraremos para implementar mudanças sociais ?

\section{BIBLIOGRAFIA}

Gérvas J, Pérez Fernández M. (1997). Las hiperlipemias y la prevención primaria de la cardiopatía isquémica. Med Clin (Barc) 109. 549-52.

Gérvas J, Pérez Fernández M. (2006). Uso y abuso del poder médico para definir enfermedad y factor de riesgo, en relación con la prevención cuaternaria. Gac Sanit, 20 Suppl 3. 66-71.

Gérvas J., Fernandez, M.P. (2003). Genética y prevención quaternária : el ejemplo de la hemacrotosis. Aten Primaria 2003 Jul 30; 32 (3): 158-62.

Gérvas J., Fernandez, M.P. (2005). Aventura y desventuras de los navegantes solitaros en el Mard de la Incertidumbre. Aten Primaria 2005 Feb 15; 35 (2): 95-8.

Gérvas J. (2006). Moderación en la actividad médica preventiva e curativa: cuatro ejemplos de necessidad de prevención quaternária en España. Gac Sanit 2006 Mar; 20 Supl 1: 127-34

Almeida, L.M. (2005). Da prevenção primordial à prevenção quaternária. Revista Portuguesa de Saúde Pública, 23, 91-6.

Starfield B. (2002). Atenção primária: equilíbrio entre necessidades de saúde, serviços e tecnologia. 1a Ed. Brasília: Organização das Nações Unidas para a Educação, a Ciência e a Cultura/Ministério da Saúde.

Zola,I. K.(1973).Pathways to the doctor-from person to patient.Social Science \& Medicine, 7, 677689.

\section{Masse}

Andrade SM, Soares DA, Braga GP. Moreira JH, Botelho FMN. Comportamentos de risco para acidentes de trânsito: um inquérito entre estudantes de medicina na Região Sul do Brasil. Rev Assoc Méd Bras 2003; 49:439-44.

Crawford, R. (1980), Healthism and the Medicalization of Everyday Life, International Journal of Health Services, 10 (3), pp. 365-388.

Chrysanthou, M (2002), Transparency and Selfhood: Utopia and the Informed Body, Social Science \& Medicine, 54 (3), pp. 469-479.

Davies J. McCrae BP, Frank J. Dochnahl A, Pickering T, Harrison B, et al. Identifying male college students' perceived health needs, barriers to seeking help, and recommendations to help men adopt healthier lifestyles. J Am Coll Health 2000; 48:259-67.

Dean, K. (1981). Self-care responses to illness: a selected review. Social Sciences \& Medicine, vol.15A, pp.673-687

Dupuy, J.P., Karsenty, S. (1974). A invasão farmacêutica. Lisboa, Editorial Presença.

Gérvas J., Fernandez, M.P. (2003). Genética y prevención quaternária : el ejemplo de la hemacrotosis. Aten Primaria 2003 Jul 30; 32 (3): 158-62.

Gérvas J., Fernandez, M.P. (2005). Aventura y desventuras de los navegantes solitaros en el Mard de la Incertidumbre. Aten Primaria 2005 Feb 15; 35 (2): 95-8.

Gérvas J. (2006). Moderación en la actividad médica preventiva e curativa: cuatro ejemplos de necessidad de prevención quaternária en España. Gac Sanit 2006 Mar; 20 Supl 1: 127-34. 
Horne, R.; Weinman, J. \& Hankíns, M. (1997). .The beliefs about medicines questionnaire: the development and evaluation of a new method for assessing the cognitive presentation of medication. Psychology and Health. Vol. , pp. 1-24. Harwood Academic Publishers.

Lowry R, Galuska DA, Fulton JE,Wechsler H, Kann L, Collins JL. Physical activity, food choiee, and weight management goals and practices among U.S. college students. Am J Prev Med 2000; 18:18-27.

Lowenberg, S., Davis, F. (1994). Beyond medicalization - demedicalization: the case of holistic health. Sociology of Health and IIness, vol.16, n 5, pp.579-599

Lupton, D. (1996). Food, the body and the self. London, Sage.

Morgan, M. (1996). Perceptions and use of anti-hipertensive drugs among cultural groups. In Williams, S.J., Calnan, M. (orgs), Modern Medicine, lay perspectives and experiences. London, UCL Press, pp. 95-116.

Ribeiro, JLP. (2005). 0 importante é a Saúde. Fundação Merck, Sharp \& Dome.

Silva,AS; Pinto,]M. (1986). Metodologia das ciências sociais. Porto, Edições Afrontamento.

Vuckovic, N.; Nichter, M. (1997). Changing patterns of pharmaceutical practice in the United States. Social Sciences \& Medicine,,vol.4,n 9,,pp.1285-1302 
